\title{
Successful Conversion Surgery for Advanced Gastric Cancer With Multiple Liver Metastases Following Ramucirumab Plus Paclitaxel Combination Treatment
}

\author{
TSUTOMU NAMIKAWA ${ }^{1}$, AKIRA MARUI ${ }^{1}$, KEIICHIRO YOKOTA $^{1}$, YUKI FUJIEDA ${ }^{1}$, \\ MASAYA MUNEKAGE ${ }^{1}$, SUNAO UEMURA ${ }^{1}$, HIROMICHI MAEDA ${ }^{1}$, HIROYUKI KITAGAWA ${ }^{1}$, \\ MICHIYA KOBAYASHI ${ }^{2}$ and KAZUHIRO HANAZAKI ${ }^{1}$ \\ ${ }^{1}$ Department of Surgery, Kochi Medical School, Kochi, Japan; \\ ${ }^{2}$ Department of Human Health and Medical Sciences, Kochi Medical School, Kochi, Japan
}

\begin{abstract}
Aim: To present the case of a patient with unresectable gastric cancer showing a remarkable effect by second-line drug treatment with ramucirumab plus paclitaxel and conversion surgery. Case Report: A 68-year-old woman who was diagnosed with gastric cancer was referred to us. Esophagogastroduodenoscopy showed an ulcerated lesion with an irregular nodulated border in the lower third of the stomach, and histology of biopsied specimens indicated a poorly differentiated adenocarcinoma. Enhanced computed tomography revealed extensive invasion of the liver, and the patients was treated using $S-1$ plus oxaliplatin as first-line chemotherapy. Because she developed liver metastases, the treatment regimen was changed to ramucirumab plus paclitaxel as the second-line treatment. After four cycles of weekly paclitaxel with ramucirumab treatment, the liver metastases had completely disappeared. Because no other metastatic lesions in other organs were detected, we performed total gastrectomy with D2 lymphadenectomy. The macroscopic findings of the surgically resected specimen revealed an ulcerated lesion with an irregularly modulated lesion measuring $9.5 \times 4.5 \mathrm{~cm}$. Pathological analysis demonstrated a poorly differentiated adenocarcinoma in the stomach, with invasion to the liver through the serosal layer, and seven lymph node metastases. The postoperative course was unremarkable, and she received ramucirumab in
\end{abstract}

This article is freely accessible online.

Correspondence to: Tsutomu Namikawa, Department of Surgery, Kochi Medical School, Kohasu, Oko-cho, Nankoku, Kochi 7838505, Japan. Tel: +81 888802370, Fax: +81 888802371, e-mail: tsutomun@kochi-u.ac.jp

Key Words: Advanced gastric cancer, unresectable metastases, conversion treatment, second line treatment. combination with paclitaxel treatment. However, liver metastasis appeared at 4 months after the operation, for which she was treated with irinotecan. Although the patient continued to receive irinotecan chemotherapy for 10 months, her general condition gradually deteriorated, and she was started on best supportive care 13 months after conversion surgery. Conclusion: Conversion surgery may prolong survival not only through first-line but also second-line treatments in selected patients with unresectable advanced gastric cancer; however, assessments of additional cases and further studies are required to establish this treatment strategy.

Gastric cancer remains one of the most common gastrointestinal malignancies, and is the second leading cause of cancer-related mortality worldwide (1). In the Japanese gastric cancer treatment guidelines, systemic drug treatment using anticancer agents or molecular targeted agents is recommended for patients with unresectable advanced and recurrent gastric cancer, with preservation of major organ function (2). Recent advances in systemic drug treatment have resulted in these therapies becoming the standard treatment for prolonging survival and have dramatically affected the prognostic impact of unresectable metastatic gastric cancer (3).

In the past few years, several investigators focused on the efficacy of surgical interventions in patients initially diagnosed with unresectable gastric cancer who were then able to undergo surgical resection after systemic drug treatment (4-8). Conversion surgery is a challenging treatment in an attempt at curative resection after downstaging or disease control obtained by use of systemic treatment (9). However, conversion surgery after second- or third-line systemic treatment is generally a difficult strategy, while conversion surgery following first-line systemic treatment did result in some survival benefit in selected patients $(10,11)$. 

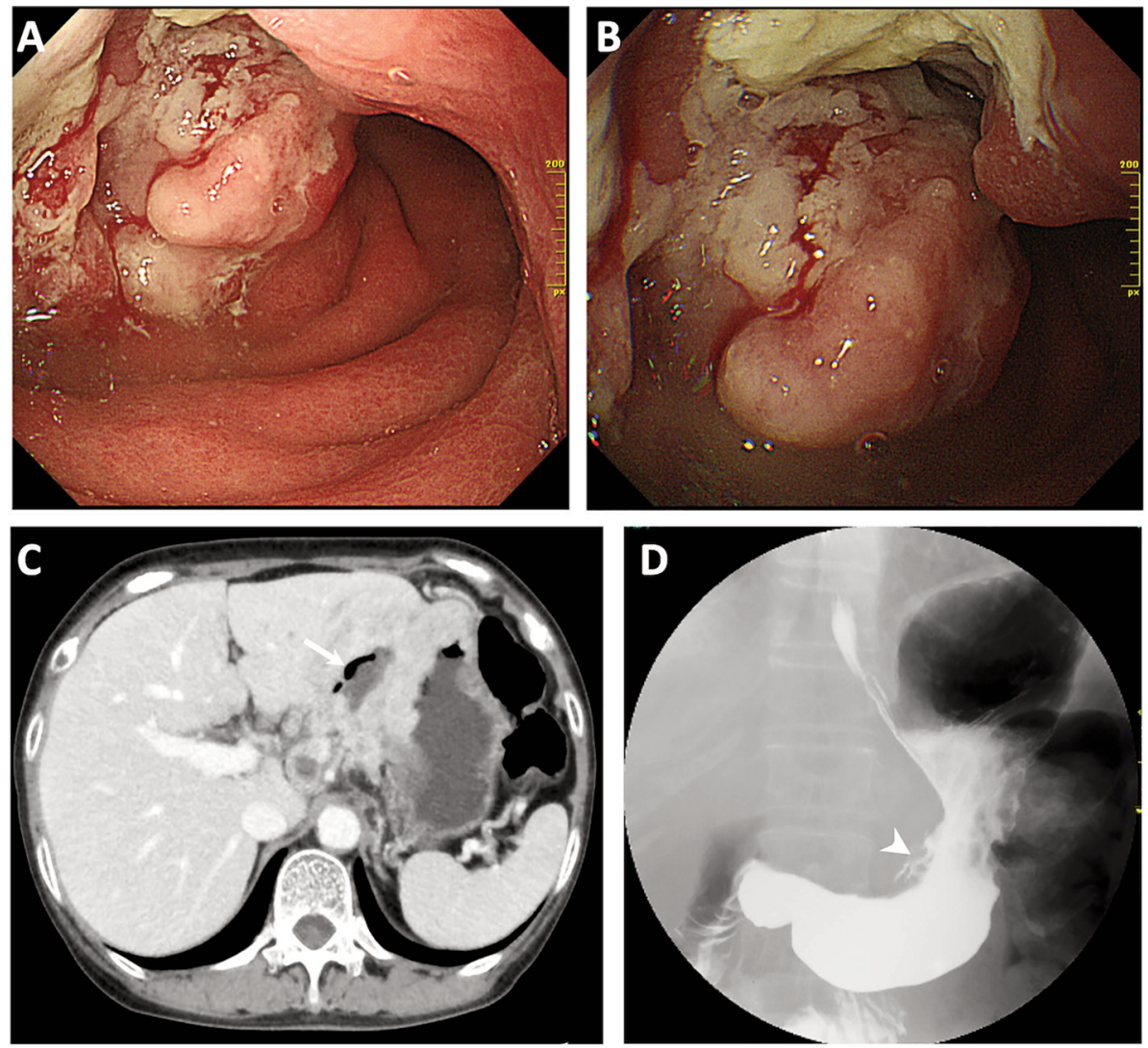

Figure 1. Initial esophagogastroduodenoscopy, computed tomography, and double-contrast upper gastrointestinal imaging. Esophagogastroduodenoscopy revealed an obviously elevated lesion with a central depression in the middle third of the stomach (A and B). Abdominal contrast-enhanced computed tomography showed thickening of the gastric wall and fluid retention with air density in the perigastric area under the lateral segment of the liver $(C$, arrow). Fluoroscopy of the upper gastrointestinal tract showed a deformity of the gastric contour, poor extension of the gastric wall, and leakage of contrast agent into the perigastric area on the lesser curvature side $(D$, arrowhead).

Herein, we present a case of patient with advanced gastric cancer who underwent conversion surgery after successful tumor downgrade by systemic second-line treatment using ramucirumab plus paclitaxel.

\section{Case Report}

A 68-year-old woman with a complaint of appetite loss and body weight presented to our Department for further evaluation after a diagnosis of gastric cancer by her local hospital. Her blood analysis showed the following: Reduced red blood cell count $\left(355 \times 10^{4} / \mathrm{mm}^{3}\right.$; normal range: $\left.386-492 \times 10^{4} / \mathrm{mm}^{3}\right)$, elevated white blood cell count $\left(10.6 \times 10^{3} / \mathrm{mm}^{3}\right.$; normal range: $\left.3.3-8.6 \times 10^{3} / \mathrm{mm}^{3}\right)$, and high C-reactive protein level $(5.5$ $\mathrm{mg} / \mathrm{dl}$; normal range: $<0.14 \mathrm{mg} / \mathrm{dl})$. A high level of the serum tumor marker carbohydrate antigen-125 (CA125) (48.0 U/ml; normal range: $<35 \mathrm{U} / \mathrm{ml}$ ) was confirmed, and levels of the serum tumor markers carcinoembryonic antigen, CA19-9 and alpha-fetoprotein were within the normal limits.
Esophagogastroduodenoscopy revealed an irregular ulcerated lesion with a nodulated border in the lower third of the stomach (Figure 1A and B). The pathological examination for biopsied specimens of the lesion demonstrated a poorly differentiated adenocarcinoma, and immunohistochemical examination of the tumor revealed negative reactivity for human epidermal growth factor receptor 2 (HER2). Abdominal contrast-enhanced computed tomography showed extensive wall thickening with heterogeneous enhancement on the lesser curvature of the stomach, with lymph nodes swelling around the stomach, and an obscured boundary between the stomach and liver, with a low-density lesion in the area between the stomach and the liver (Figure 1C). Upper gastrointestinal imaging using a water-soluble contrast agent revealed a deformity of the gastric contour with poor extension and leakage of the contrast agent into the perigastric area on the lesser curvature side (Figure 1D).

We diagnosed cT4N2M0, stage IV gastric cancer accompanied by liver invasion according to the Japanese 

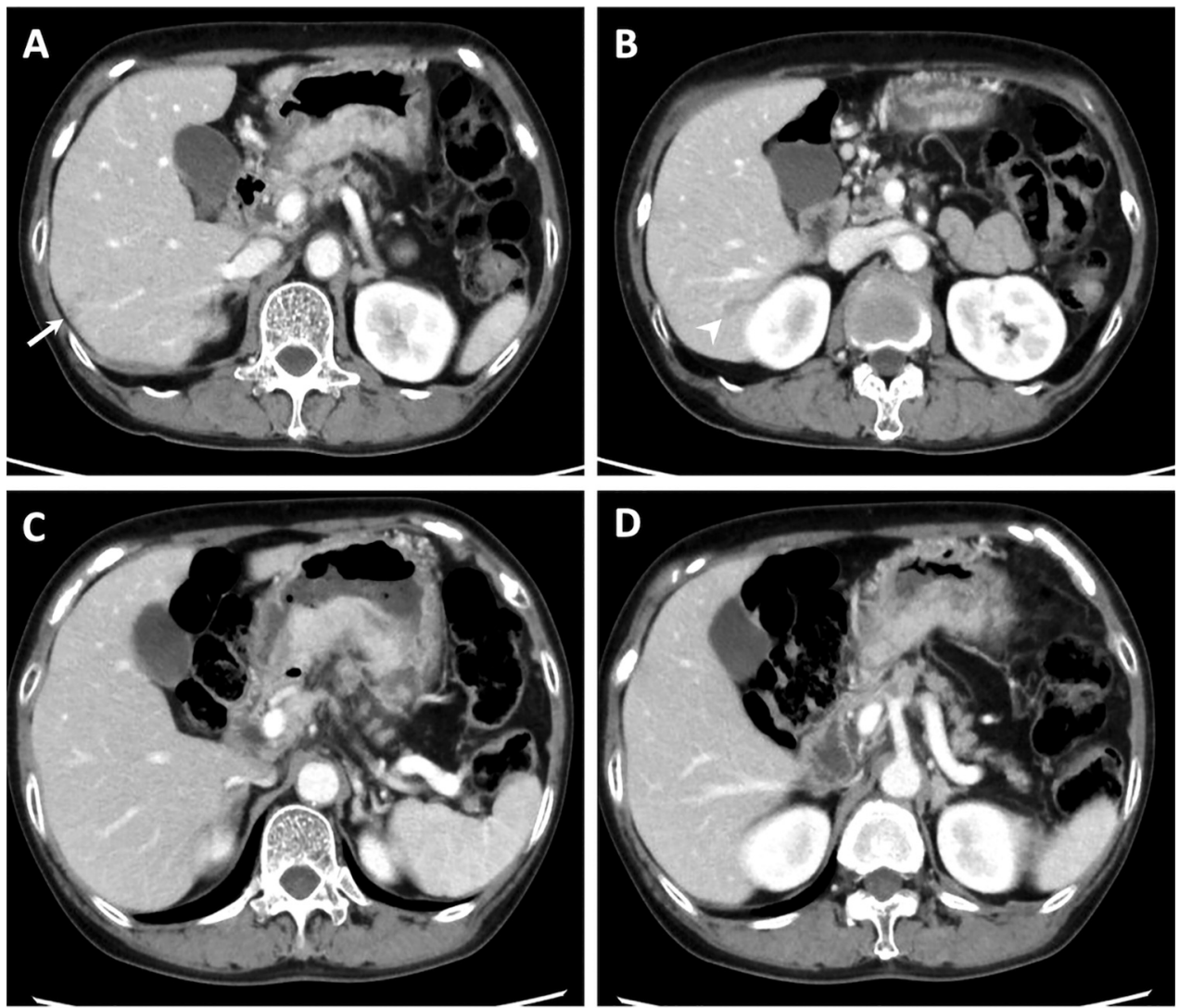

Figure 2. Abdominal contrast-enhanced computed tomography before ( $A$ and $B)$ and after $(C$ and $D)$ systemic treatment using ramucirumab plus paclitaxel. Abdominal contrast-enhanced computed tomography before ramucirumab plus paclitaxel treatment showed liver metastases (A, arrow; $B$, arrowhead). After four cycles of weekly paclitaxel with ramucirumab treatment, the liver metastases completely disappeared.

classification system (12), treating the patient with S-1 plus oxaliplatin as the first-line chemotherapy. S-1 was administered at $80 \mathrm{mg} / \mathrm{m}^{2}$ body surface area per day for 2 weeks, followed by 1 week of no chemotherapy. On day 1 of the 21-day cycle, the patient was also intravenously administered $100 \mathrm{mg} / \mathrm{m}^{2}$ per day of oxaliplatin. After four courses of chemotherapy, liver metastases were detected on abdominal contrast-enhanced computed tomography (Figure $2 \mathrm{~A}$ and $\mathrm{B})$. Therefore, the regimen was changed to ramucirumab in combination with paclitaxel chemotherapy as the second-line treatment. After four cycles of weekly paclitaxel with ramucirumab, the liver metastases had completely disappeared (Figure 2C and D).

Since enhanced computed tomography showed no further metastatic lesions in any other organs, conversion surgery was planned. Intraoperatively, there were dense adhesions with sclerotic tissue changes between the liver and stomach, and we performed total gastrectomy with D2 lymphadenectomy followed by Roux-en-Y reconstruction. The operative time was $312 \mathrm{~min}$, and the estimated blood loss was $450 \mathrm{ml}$.
The macroscopic findings of the surgically resected specimen revealed an ulcerated lesion with an irregularly modulated lesion measuring $9.5 \times 4.5 \mathrm{~cm}$ in the middle third of the stomach (Figure 3A). Pathological analysis of the specimen demonstrated a poorly differentiated adenocarcinoma in the stomach, with invasion to the liver through the serosal layer and seven lymph node metastases (Figure 3B). The tumor after preoperative therapy was classified as ypT4N3M0 stage IIIC, and the histological response after preoperative therapy was grade 1a according to the Japanese classification system (12).

The patient was discharged on postoperative day 14 without complications, and she received ramucirumab in combination with paclitaxel treatment. However, the patient developed liver metastasis at 4 months after the operation, for which she was started on treatment with irinotecan. Despite a 10-month course of irinotecan chemotherapy, her general condition gradually deteriorated, and her treatment plan was changed to best supportive care 13 months after conversion surgery. 

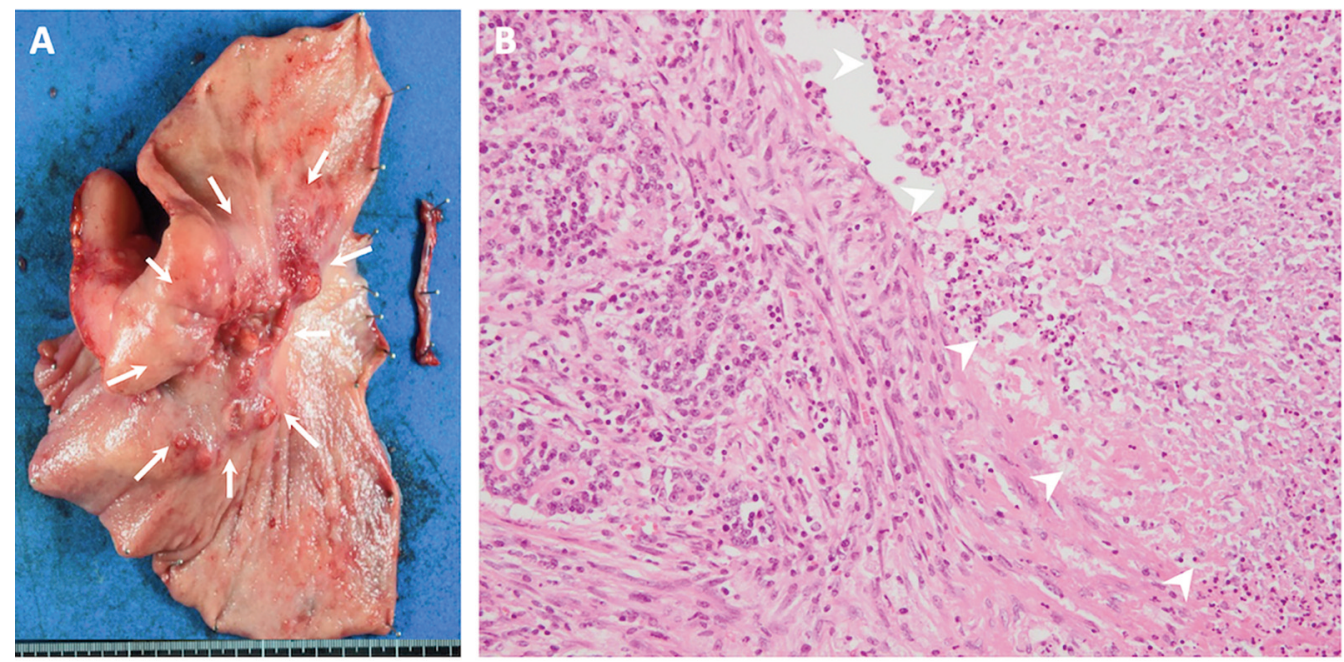

Figure 3. The gross appearance and pathological examination of the surgically resected specimen. The macroscopic appearance of the resected specimen revealed an ulcerated tumor, with an irregularly modulated lesion measuring $9.5 \times 4.5 \mathrm{~cm}$ in the middle third of the stomach (A, arrows). Hematoxylin and eosin-stained sections of the gastric tumor demonstrated serosal invasion of a poorly differentiated adenocarcinoma with necrotic change (B, arrowheads).

\section{Discussion}

We herein describe the case of a patient with initially unresectable advanced gastric cancer who underwent conversion surgery after the control of distant metastases by second-line systemic treatment with ramucirumab plus paclitaxel. We analyzed conversion surgery for gastric cancer after second-line systemic treatment reported from 2000 and 2021 in the English literature as found in a search of the Medline and PubMed databases using the key words "unresectable gastric cancer", "second- or third-line treatment" and "conversion surgery." Articles published only in abstract form were excluded, and we obtained clinicopathological data for each patient. To the best of our knowledge, this is only the third reported case for which the conversion surgery after second-line systemic treatment for initially unresectable advanced gastric cancer is described in the English literature.

Table I lists the clinicopathologic characteristics of the two previously reported cases $(13,14)$ and the present case. The median age of the patients was 68 (range $=68-77$ ) years, and the male-to-female ratio was 1:2. Gastric tumor in the middle third of the stomach was reported in all cases, and the macroscopic type was also ulcerated lesions. The firstline regimen for systemic treatment was $\mathrm{S}-1$ plus cisplatin for one patient and S-1 plus oxaliplatin for the other two. The second-line systemic treatment was ramucirumab plus paclitaxel for all patients. Conversion surgery was performed after second-line treatment in two patients and after thirdline treatment in one. Treatment consisted of total gastrectomy in one patient and distal gastrectomy in two.
Histological examinations of the gastric adenocarcinomas showed two intestinal types and one diffuse-type carcinoma.

According to the Japanese guidelines for gastric cancer, $\mathrm{S}-1$ plus cisplatin is the recommended systemic treatment as first-line chemotherapy for patients with HER2-negative disease $(2,15,16)$. S-1 plus oxaliplatin is also recommended chemotherapy regimen for unresectable advanced gastric cancer and was proven to be effective in pivotal trials (1720). Recent developments in second-line and further treatments, such as ramucirumab, nivolumab, and trifluridine/tipiracil, for metastatic gastric cancer have improved overall survival and quality of life (21-23). In particular, the recent advances in newly developed molecular targeted drugs, such as trastuzumab, ramucirumab, and nivolumab combined with chemotherapy, have had a significant effect on improving the prognosis in patients with initially unresectable advanced gastric cancer $(21,22)$.

Several investigators demonstrated that the survival of patients who were able to undergo conversion surgery was longer than those who continued chemotherapy $(8,10,24)$. These studies have shown the practical benefits of conversion surgery following first-line systemic treatment in selected patients. However, it remains unclear whether conversion surgery after second-line treatment is an effective strategy, following which a patient's condition generally deteriorates and often worsens due to exhaustion.

Ramucirumab is a molecular targeted angiogenesis inhibitor, that is a humanized monoclonal antibody directed against vascular endothelial growth factor receptor-2 $(21,25)$. Considering its characteristics as an angiogenesis inhibitor and the circulating half-life of this drug, a more than 28-day 


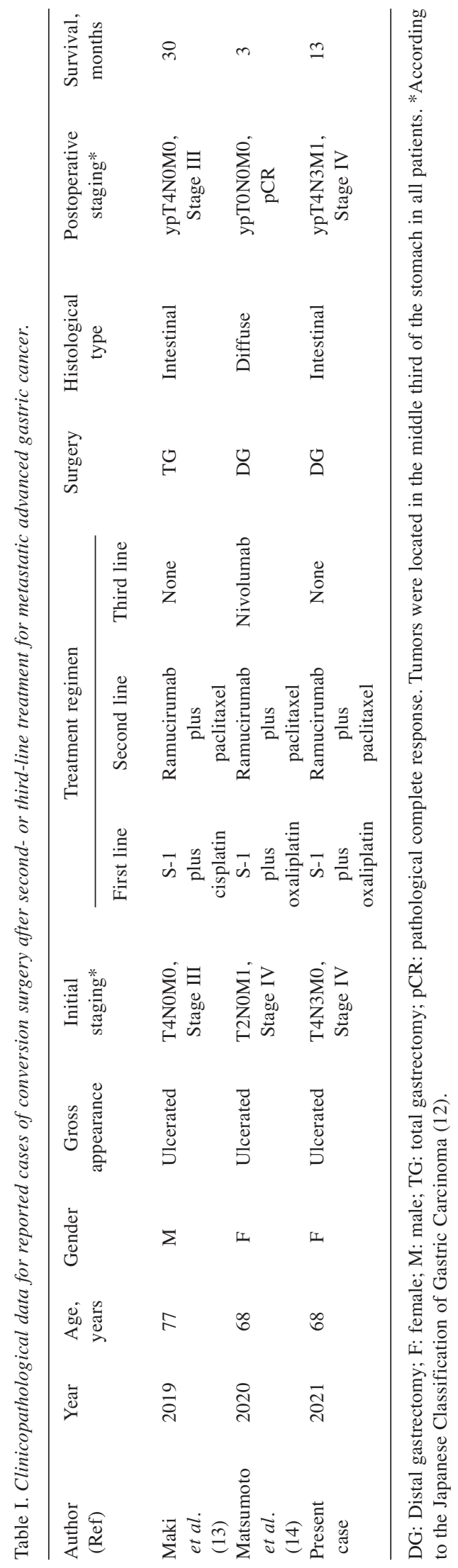

interval between the last administration and an operation is recommended when surgery is planned (14). In the present case, the interval between the last administration of ramucirumab and surgery was set at 30 days. These considerations may also be needed to minimize the occurrence of complications associated with conversion surgery.

Yoshida et al. classified stage IV gastric cancer into four categories for conversion surgery on the basis of the biology and heterogeneous characteristics of the tumor (9). According to this new therapeutic classification, the present case was classified as category 2, which was a marginally resectable metastasis, and the operation would not be considered to be the best choice for initial treatment. Yamaguchi et al. reported that among 135 patients with gastric cancer classified as category 2 , the median survival was significantly longer in patients who underwent conversion surgery than in those without surgery (30.5 months vs. 11.0 months; $p<0.001)$ (8). However, the preoperative determination of whether curative resection with negative resection margins is possible remains clinically challenging for patients with category 2 isease $(8,9)$.

Previous studies reported that additional systemic treatment should be continued even if conversion therapy can be performed with curative resection $(8,9,17,26)$. The present patient developed liver metastasis at 4 months after the operation despite the continuation of postoperative ramucirumab plus paclitaxel treatment. Further assessments of additional cases are required to determine the benefit of conversion surgery following second- or third-line systemic treatment.

In conclusion, conversion surgery after second- or thirdline systemic treatment might be a treatment option for patients with initially unresectable gastric cancer, even if first-line treatment fails to improve treatment outcomes. Although prospective randomized control studies are unrealistic due to the small number of patients, further investigations, including case-control studies with large sample sizes, are required to validate the use of conversion surgery following late line systemic treatment.

\section{Conflicts of Interest}

None declared.

\section{Authors' Contributions}

T. Namikawa, A. Marui, K. Yokota and Y. Fujieda performed the surgical procedure; T. Namikawa and H. Maeda reviewed literature data; T. Namikawa, M. Munekage, S. Uemura and H. Kitagawa performed preoperative investigation the patient; T. Namikawa prepared the draft of the article; M. Kobayashi was advisor of the surgical procedures; T. Namikawa and K. Hanazaki reviewed the final version of the article. All Authors read and approved the final version of the article. 


\section{References}

1 Siegel RL, Miller KD, Fuchs HE and Jemal A: Cancer statistics, 2021. CA Cancer J Clin 71(1): 7-33, 2021. PMID: 33433946. DOI: $10.3322 /$ caac. 21654

2 Japanese Gastric Cancer Association: Japanese gastric cancer treatment guidelines 2018 (5th edition). Gastric Cancer 24(1): 121, 2021. PMID: 32060757. DOI: 10.1007/s10120-020-01042-y

3 Namikawa T, Munekage E, Munekage M, Maeda H, Yatabe T, Kitagawa H, Kobayashi M and Hanazaki K: Evaluation of systemic inflammatory response biomarkers in patients receiving chemotherapy for unresectable and recurrent advanced gastric cancer. Oncology 90(6): 321-326, 2016. PMID: 27225990. DOI: $10.1159 / 000446373$

4 Mieno H, Yamashita K, Hosoda K, Moriya H, Higuchi K, Azuma M, Komori S, Yoshida T, Tanabe S, Koizumi W, Katada $\mathrm{N}$ and Watanabe $\mathrm{M}$ : Conversion surgery after combination chemotherapy of docetaxel, cisplatin and S-1 (DCS) for faradvanced gastric cancer. Surg Today 47(10): 1249-1258, 2017. PMID: 28365892. DOI: 10.1007/s00595-017-1512-Z

5 Kinoshita J, Fushida S, Tsukada T, Oyama K, Okamoto K, Makino I, Nakamura K, Miyashita T, Tajima H, Takamura H, Ninomiya I and Ohta T: Efficacy of conversion gastrectomy following docetaxel, cisplatin, and S-1 therapy in potentially resectable stage IV gastric cancer. Eur J Surg Oncol 41(10): 13541360, 2015. PMID: 26028256. DOI: 10.1016/j.ejso.2015.04.021

6 Terashima M: Conversion therapy for gastric cancer: who can make conversion as successful as Goromaru? Gastric Cancer 19(3): 685-686, 2016. PMID: 27055560. DOI: 10.1007/s10120016-0609-1

7 Fukuchi M, Mochiki E, Ishiguro T, Ogura T, Sobajima J, Kumagai Y, Ishibashi $\mathrm{K}$ and Ishida H: Efficacy of conversion surgery following S-1 plus cisplatin or oxaliplatin chemotherapy for unresectable gastric cancer. Anticancer Res 37(3): 13431347, 2017. PMID: 28314301. DOI: 10.21873/anticanres.11453

8 Yamaguchi K, Yoshida K, Tanahashi T, Takahashi T, Matsuhashi $\mathrm{N}$, Tanaka Y, Tanabe K and Ohdan H: The long-term survival of stage IV gastric cancer patients with conversion therapy. Gastric Cancer 21(2): 315-323, 2018. PMID: 28616743. DOI: 10.1007/ s10120-017-0738-1

9 Yoshida K, Yamaguchi K, Okumura N, Tanahashi T and Kodera Y: Is conversion therapy possible in stage IV gastric cancer: the proposal of new biological categories of classification. Gastric Cancer 19(2): 329-338, 2016. PMID: 26643880. DOI: 10.1007/ s10120-015-0575-Z

10 Fukuchi M, Mochiki E, Ishiguro T, Kumagai Y, Ishibashi K and Ishida $\mathrm{H}$ : Prognostic significance of conversion surgery following first- or second-line chemotherapy for unresectable gastric cancer. Anticancer Res 38(11): 6473-6478, 2018. PMID: 30396974. DOI: 10.21873/anticanres.13010

11 Namikawa T, Tsuda S, Fujisawa K, Iwabu J, Uemura S, Tsujii $\mathrm{S}$, Maeda H, Kitagawa H, Kobayashi $\mathrm{M}$ and Hanazaki K: Conversion surgery after S-1 plus oxaliplatin combination chemotherapy for advanced gastric cancer with multiple liver metastases. Clin J Gastroenterol 11(4): 297-301, 2018. PMID: 29500608. DOI: $10.1007 / \mathrm{s} 12328-018-0842-8$

12 Japanese Gastric Cancer Association: Japanese classification of gastric carcinoma: 3rd English edition. Gastric Cancer 14(2): 101-112, 2011. PMID: 21573743. DOI: 10.1007/s10120-0110041-5
13 Maki H, Yuasa Y, Fujiwara S, Fukuta M, Takeuchi T, Fujimoto $\mathrm{K}$, Tsuneki T, Matsuo Y, Mori O, Eto S, Tomibayashi A and Ishikura $\mathrm{H}$ : Unresectable advanced gastric cancer with skin invasion followed by total gastrectomy after second-line chemotherapy. Case Rep Oncol 12(2): 671-680, 2019. PMID: 31572157. DOI: $10.1159 / 000502415$

14 Matsumoto R, Arigami T, Matsushita D, Okubo K, Tanaka T, Yanagita S, Sasaki K, Noda M, Kita Y, Mori S, Kurahara H and Ohtsuka T: Conversion surgery for stage IV gastric cancer with a complete pathological response to nivolumab: a case report. World J Surg Oncol 18(1): 179, 2020. PMID: 32693806. DOI: 10.1186/s12957-020-01954-0

15 Namikawa T, Shiga M, Ichikawa K, Kitagawa H, Kobayashi M and Hanazaki K: Metachronous liver and bone metastasis from small early gastric carcinoma without lymph node involvement: A case report. Mol Clin Oncol 1(2): 249-252, 2013. PMID: 24649155. DOI: $10.3892 / \mathrm{mco} .2012 .50$

16 Namikawa T, Fukudome I, Ogawa M, Munekage E, Munekage M, Shiga M, Maeda H, Kitagawa H, Kobayashi M and Hanazaki $\mathrm{K}$ : Clinical efficacy of protein-bound polysaccharide $\mathrm{K}$ in patients with gastric cancer undergoing chemotherapy with an oral fluoropyrimidine (S-1). Eur J Surg Oncol 41(6): 795-800, 2015. PMID: 25887287. DOI: 10.1016/j.ejso.2015.02.012

17 Yamada Y, Higuchi K, Nishikawa K, Gotoh M, Fuse N, Sugimoto N, Nishina T, Amagai K, Chin K, Niwa Y, Tsuji A, Imamura H, Tsuda M, Yasui H, Fujii H, Yamaguchi K, Yasui H, Hironaka S, Shimada K, Miwa H, Hamada C and Hyodo I: Phase III study comparing oxaliplatin plus S-1 with cisplatin plus S-1 in chemotherapy-naïve patients with advanced gastric cancer. Ann Oncol 26(1): 141-148, 2015. PMID: 25316259. DOI: $10.1093 /$ annonc/mdu472

18 Nishikawa K, Yamada Y, Ishido K, Gotoh M, Bando H, Sugimoto N, Nishina T, Amagai K, Chin K, Niwa Y, Tsuji A, Imamura H, Tsuda M, Yasui H, Fujii H, Yamaguchi K, Yasui H, Hironaka S, Shimada K, Miwa H, Hamada C and Hyodo I: Impact of progression type on overall survival in patients with advanced gastric cancer based on randomized phase III study of S-1 plus oxaliplatin versus S-1 plus cisplatin. Gastric Cancer 20(4): 640-645, 2017. PMID: 27822684. DOI: 10.1007/s10120016-0666-5

19 Koizumi W, Takiuchi H, Yamada Y, Boku N, Fuse N, Muro K, Komatsu Y and Tsuburaya A: Phase II study of oxaliplatin plus S-1 as first-line treatment for advanced gastric cancer (G-SOX study). Ann Oncol 21(5): 1001-1005, 2010. PMID: 19875759. DOI: 10.1093/annonc/mdp464

20 Terashima M, Iwasaki Y, Mizusawa J, Katayama H, Nakamura K, Katai H, Yoshikawa T, Ito Y, Kaji M, Kimura Y, Hirao M, Yamada M, Kurita A, Takagi M, Boku N, Sano T, Sasako M and Stomach Cancer Study Group, Japan Clinical Oncology Group: Randomized phase III trial of gastrectomy with or without neoadjuvant S-1 plus cisplatin for type 4 or large type 3 gastric cancer, the short-term safety and surgical results: Japan Clinical Oncology Group Study (JCOG0501). Gastric Cancer 22(5): 1044-1052, 2019. PMID: 30827001. DOI: 10.1007/s10120-01900941-z

21 Tokunaga M, Sato Y, Nakagawa M, Aburatani T, Matsuyama T, Nakajima Y and Kinugasa Y: Perioperative chemotherapy for locally advanced gastric cancer in Japan: current and future perspectives. Surg Today 50(1): 30-37, 2020. PMID: 31612329. DOI: $10.1007 / \mathrm{s} 00595-019-01896-5$ 
22 Wilke H, Muro K, Van Cutsem E, Oh SC, Bodoky G, Shimada Y, Hironaka S, Sugimoto N, Lipatov O, Kim TY, Cunningham D, Rougier P, Komatsu Y, Ajani J, Emig M, Carlesi R, Ferry D, Chandrawansa K, Schwartz JD, Ohtsu A and RAINBOW Study Group: Ramucirumab plus paclitaxel versus placebo plus paclitaxel in patients with previously treated advanced gastric or gastro-oesophageal junction adenocarcinoma (RAINBOW): a double-blind, randomised phase 3 trial. Lancet Oncol 15(11): 1224-1235, 2014. PMID: 25240821. DOI: 10.1016/S14702045(14)70420-6

23 Kang YK, Boku N, Satoh T, Ryu MH, Chao Y, Kato K, Chung HC, Chen JS, Muro K, Kang WK, Yeh KH, Yoshikawa T, Oh SC, Bai LY, Tamura T, Lee KW, Hamamoto Y, Kim JG, Chin K, Oh DY, Minashi K, Cho JY, Tsuda M and Chen LT: Nivolumab in patients with advanced gastric or gastro-oesophageal junction cancer refractory to, or intolerant of, at least two previous chemotherapy regimens (ONO-4538-12, ATTRACTION-2): a randomised, double-blind, placebo-controlled, phase 3 trial. Lancet 390(10111): 2461-2471, 2017. PMID: 28993052. DOI: $10.1016 /$ S0140-6736(17)31827-5

24 Shitara K, Doi T, Dvorkin M, Mansoor W, Arkenau HT, Prokharau A, Alsina M, Ghidini M, Faustino C, Gorbunova V, Zhavrid E, Nishikawa K, Hosokawa A, Yalçın Ş, Fujitani K, Beretta GD, Cutsem EV, Winkler RE, Makris L, Ilson DH and Tabernero J: Trifluridine/tipiracil versus placebo in patients with heavily pretreated metastatic gastric cancer (TAGS): a randomised, double-blind, placebo-controlled, phase 3 trial. Lancet Oncol 19(11): 1437-1448, 2018. PMID: 30355453. DOI: $10.1016 /$ S1470-2045(18)30739-3
25 Sato Y, Ohnuma H, Nobuoka T, Hirakawa M, Sagawa T, Fujikawa K, Takahashi Y, Shinya M, Katsuki S, Takahashi M, Maeda M, Okagawa Y, Naoki U, Kikuch S, Okamoto K, Miyamoto H, Shimada M, Takemasa I, Kato J and Takayama T: Conversion therapy for inoperable advanced gastric cancer patients by docetaxel, cisplatin, and S-1 (DCS) chemotherapy: a multi-institutional retrospective study. Gastric Cancer 20(3): 517-526, 2017. PMID: 27553665. DOI: 10.1007/s10120-0160633-1

26 Uehata N, Kouzu K, Tsujimoto H, Sugasawa H, Wakamatsu K, Kishi $\mathrm{Y}$ and Ueno H: Long-term survival achieved by repeated administration of ramucirumab after drug holidays due to proteinuria in recurrent gastric cancer. Surg Case Rep 7(1): 97, 2021. PMID: 33876326. DOI: 10.1186/s40792-021-01185-9

Received May 15, 2021

Revised June 11, 2021

Accepted June 15, 2021 